\title{
DESIGN OF A RELIABLE PROCESSING PIPELINE FOR THE NON-INTRUSIVE MEASUREMENT OF FEET TRAJECTORIES WITH LASERS
}

\author{
S. Piérard, S. Azrour, and M. Van Droogenbroeck \\ INTELSIG Laboratory, Montefiore Institute, University of Liège, Belgium
}

\begin{abstract}
Reliable measurements of feet trajectories are needed in some applications, such as biomedical applications. This paper describes the data processing pipeline used in GAIMS, which is a non-intrusive system that measures feet trajectories based on multiple range laser scanners. Our processing pipeline relies on a new tracking paradigm, and it is based on two innovative algorithms: the first algorithm localizes the feet directly from the observed point cloud without any clustering, and the other algorithm identifies the feet. After reviewing the various types of noise affecting the point cloud, this paper explains the limitations of the classical processing approach and gives an overview of our new pipeline. The effectiveness of the proposed approach is established by discussing the results that have been obtained in several studies based on GAIMS.
\end{abstract}

Index Terms-GAIMS, feet, tracking, multiple sclerosis, biometric identification

\section{INTRODUCTION}

Interesting information about the gait of an observed person can be derived from his feet trajectories. Measuring them can be useful in many different high level applications such as the biometric identification [6,37], the follow-up of patients with some neurological diseases [1, 5, 32], etc. For the most applications, non-intrusive measuring systems are necessary or preferable. In a non-intrusive system, the observed person does not need to carry any form of a tag, such as sensors, markers, special clothes, etc. In our previous works $[31,32]$, we have introduced the non-intrusive system GAIMS (GAIt Measuring System) which measures feet trajectories by combining and analyzing the distance profiles measured by several range laser scanners (RLSs) covering a common horizontal plane located a few centimeters above the floor (see Figure 1). This system tracks the feet both in the swing and stance phases, and can be used in large areas. Moreover, it is nearly insensitive to ambient lighting and can be used in total darkness.

The principle of RLSs is the following. They measure distances between themselves and a few points of the scene that are in line of sight. The sensor emits a light pulse (usually in the infrared band) which is reflected by the objects in the scene and sent back to a light detector, collocated with the pulse source. The distance to the hit point is derived from the time needed by the signal to travel forth and back. RLSs measure one distance at a time, but the measure is repeated at high speed and periodically in a set of directions. Assuming that the sensors are punctual, a polar to Cartesian coordinates transform suffices to compute the point cloud corresponding to the discretized distance profile that is measured.

In order to combine the information provided by several sensors, their positions and orientations must be determined. Many calibration procedures have been proposed. Piérard et al. [34] describe a

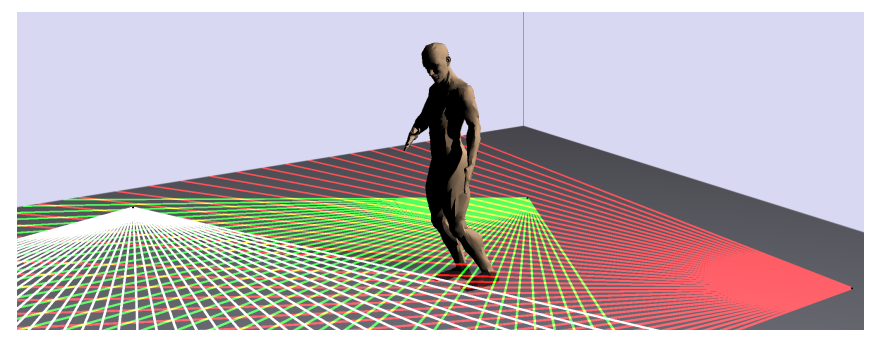

Fig. 1. GAIMS measures feet trajectories by using several range laser scanners covering a common horizontal plane located at the height of the ankles. A few laser beams are depicted for three sensors.

calibration procedure that consists in placing a known object at several places in the scene. Hashimoto et al. [17] achieve the calibration thanks to two planes placed in the environment. Zhao et al. [41] propose to align the backgrounds manually and to run an optimization algorithm, and Glas et al. [15] align the trajectories of the observed persons. After calibration, the point clouds seen by all sensors can be registered, and merged into a global point cloud. The use of several sensors allows to reduce (self-)occlusions, cover a larger area, and enhance the global point cloud density.

Most laser-based tracking works presented in the literature are related to the tracking of persons (or groups of persons [24]). Only few methods are devoted to the tracking of legs [34, 37, 39], and these works usually aim at tracking persons by fusing leg tracks [2, $8,9,38]$. It appears that obtaining a high precision and accuracy is not the main requirement for those systems, to the contrary of the applications for which GAIMS has been designed.

This paper describes the data processing pipeline used in GAIMS and explains our design choices. This document is organized as follows. Section 2 elaborates on the various kinds of noise affecting the global point cloud. Then, Section 3 explains why the classical pipeline to track objects is not suitable for our needs, and Section 4 presents an overview of the data processing pipeline proposed to overcome the limitations of the classical approaches. Finally, Section 5 summarizes the main results that have been obtained with GAIMS, and explains how our processing pipeline improves on previous works. A short conclusion is given in Section 6.

\section{NOISE AFFECTING THE GLOBAL POINT CLOUD}

The current version of GAIMS relies on 4 RLSs BEA LZR-il00 (placed in the corners of a rectangular room, as illustrated in [31]). In the following, we analyze the main sources of noise affecting the global point cloud, based on the characteristics of these sensors. Furthermore, we develop some examples to highlight the order of magnitude of these limitations. 
S. Piérard, S. Azrour, and M. VAn Droogenbroeck. Design of reliable processing pipeline for the non-intrusive measurement of feet trajectories with lasers. In IEEE International Conference on Acoustics, Speech, and Signal Processing (ICASSP), Florence, Italy, pages 4432-4436, May 2014.

Limited precision of the distance measurements. The announced distance resolution is about $1 \mathrm{~mm}$. In practice however, we observe a temporal variation of a few millimeters, and sometimes even a few centimeters, on the acquired distances. The sensors are strongly disturbed by highly reflective materials (eg metal), and black materials (in the infrared band). At discontinuities in the distance profile, the sensors produce a random distance measure between the minimum and the maximum distance (this observation is similar to the flying pixels observed by Lindner et al. [22] with $P M D$ cameras). Therefore, the sensors may see points where there is no object in the scene, and robustness to outliers is mandatory.

Low angular resolution. The sensors measure distances in 274 directions spanning a $\Theta=96.3281^{\circ}$ wide angle. The number of points seen on an object rapidly decreases when the object moves away from the sensor. Let us consider a circular object of radius $r$, whose center is located at a distance $d$ from the sensor. The sensor sees this object in an angle $\theta=2 \arcsin \left(\frac{r}{d}\right)$, and a minimum of $\left\lfloor\theta \frac{274-1}{\Theta}\right\rfloor$ points, where $\lfloor\cdot\rfloor$ denotes the "round down" operator.

Example 1. A sensor sees only 3 points on a leg (the typical size is $r=5 \mathrm{~cm}$ with trousers) located at $5 \mathrm{~m}$ from the sensor. The use of multiple sensors helps to overcome this limitation.

Scanning duration. The acquisition rate is $15 \mathrm{~Hz}$, and the scanning is obtained thanks to an internal mirror turning at $360 \times 15=$ $5400 \%$. Therefore, the infrared beam turns at $\Omega=10800 \% \mathrm{~s}$ and a complete distance profile is acquired in $\frac{\Theta}{\Omega} \simeq 9 \mathrm{~ms}$. This is important when the object is moving. Let us consider an object of radius $r$, located at a distance $d \gg r$, and turning around the sensor with a small angular velocity $\omega$. It can be showed that, due to its motion, the object is seen with an apparent radius $r^{\prime} \simeq r\left(1+\frac{\omega}{\Omega}\right)$.

Example 2. Let us consider a person walking in a direction perpendicular to the sensor's line of sight. For a walking speed of $5 \mathrm{~km} / \mathrm{h}$, the maximal speed of the feet is approximately $16 \mathrm{~km} / \mathrm{h}$. This gives $\omega=\frac{16000}{3600} \frac{1}{d} \frac{360}{2 \pi} \%$ s. The motion induces an error of only $2.36 \%$ on the perceived leg size for $d=1 \mathrm{~m}$. As this error is negligible, the selected sensors are fast enough to track feet with a high accuracy.

Asynchronism. As the manufacturer did not foresee a mean to synchronize the sensors, there may be a difference of $\frac{1}{15} s$ between the acquisition time of the points in the global point cloud. The displacement of the objects during $\frac{1}{15} s$ should be negligible in comparison to their size. Otherwise, the perceived shape of the objects would be highly altered.

Example 3. For a walking speed of $5 \mathrm{~km} / \mathrm{h}$, the maximal speed of the feet is approximately $16 \mathrm{~km} / \mathrm{h}$. Therefore, during $\frac{1}{15} \mathrm{~s}$, a foot can move by $29.6 \mathrm{~cm}$. As this is larger than the size of the element, highly deformed global point clouds are expected, and advanced processing strategies are necessary.

It follows, from this analysis, that the point cloud is distorted, even if the person does not move. When the person is walking rapidly, the asynchronism cannot be neglected, and the perceived point cloud is highly deformed (either compacted or elongated depending on the motion direction). Other reasons for distorted clouds include the limited field of view, the (self-)occlusions, and the flying pixels. We need to consider all these limitations when developing the algorithms to analyze the information provided by RLSs. In the remainder of this paper, we show that a proper processing pipeline design enables us to reach high precision and accuracy on the trajectories measured by GAIMS.

\section{WEAKNESSES OF CLASSICAL APPROACHES}

A classical processing approach to track objects based on successive point clouds starts with the segmentation of the scene into its components (the walls, the objects, the legs, etc). Afterwards, the location of each component is defined (usually by its centroid), and tracking coupled to data association techniques are used to estimate the trajectory of each component. Even if this processing flow is often encountered in the literature, it is inappropriate to measure the feet trajectories for several reasons that are summarized hereafter.

Segmentation. There are mainly two types of segmentation methods: those detecting the discontinuities in a distance profile (i.e. the breakpoints [35]) and those clustering a point cloud. Whereas the methods of the first type $[7,19,38]$ are tailored only for a single sensor, the methods of the second type take the signals measured by all RLSs into account simultaneously. Fod et al. [13] assume that the various elements in the scene are separated by at least $10 \mathrm{~cm}$, and Zhao et al. [41] group the points within a radius of $15 \mathrm{~cm}$. Clearly, this approach is inappropriate for our applications: it is difficult to separate the legs at the swing phase middle since they are very close. A similar observation has been made by Cui et al. in $[9,10,11]$. The deformation of the point cloud (explained in the previous section) may also cause difficulties for clustering-based approaches.

Localization. After the segmentation, a location has to be estimated for each blob. The centroid is considered in [38] and [41]. But, the feet motion and the sensors asynchronism cause deformed point clouds. Moreover, the points are not sampled regularly along the leg's contour because a sensor sees only one side of it [38], and also due to the (self-)occlusions in the scene. Therefore, the blob's centroid is a biased estimation of its real location, which implies some bias in the resulting feet trajectories.

Tracking. Another source of imperfection originates from the tracking, which is often done simultaneously with the noise filtering, as with the (extended) KALMAN filter [18] (see $e g$ [2, 11, 13, 17, 24, $38,41]$ ). Such a filter requires to specify a model of motion. Several models of the human walk have been described and used in the literature in order to analyze RLS data [2, 8, 10, 12, 21, 23, 25, 37, 38, 41]. The role of such a model is to help filtering out the component of the signal which does not correspond to the movement predicted by the model. Obviously, it is delicate to guarantee that the part of the gait which is specific to the observed person (that is the signal of interest for the final applications) is preserved, since one can only use a model representing the average gait of the healthy population. Moreover, the existing models are very simple (they assume a constant velocity, a constant acceleration in each phase of the gait cycle, or only a few degrees of freedom). In consequence, the precision of the tracking is affected by the deficiency in realism. For example, Chung et al. [8] have observed, with their model, errors as large as $16 \mathrm{~cm}$ on the foot displacement during only $200 \mathrm{~ms}$.

Data association. In a point cloud, there exists no information to help distinguishing between the left and the right feet. The data association is thus typically performed thanks to the tracker, based on the previous measurements. Nevertheless, in the context of people tracking inside groups, Mucientes et al. [24] have underlined the difficulty of the data association problem, one reason being the proximity of the tracked elements. In the context of feet tracking, the data association problem is amplified. Even a single data association 
S. Piérard, S. Azrour, and M. Van Droogenbroeck. Design of reliable processing pipeline for the non-intrusive measurement of feet trajectories with lasers. In IEEE International Conference on Acoustics, Speech, and Signal Processing (ICASSP), Florence, Italy, pages 4432-4436, May 2014.

\begin{tabular}{|c|}
\hline \\
global point cloud \\
registration \& merging \\
\hline polar to cartesian \\
\hline$+\downarrow$ \\
background subtraction \\
\hline
\end{tabular}

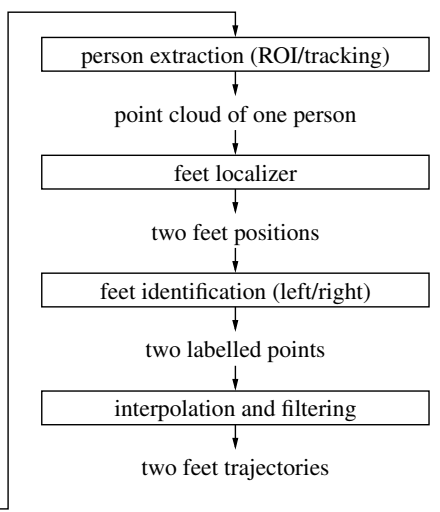

Fig. 2. The new proposed processing pipeline to measure feet trajectories with GAIMS.

failure may have severe consequences (eg for medical applications in which the movement of each foot has to be described separately) since a crossing between the two feet trajectories may occur. Moreover, bootstrapping the tracking and data association procedure is always delicate.

Simplifying assumptions. It should also be noted that many simplifying assumptions have been used in the literature. For example, Carballo et al. [7] and Xavier et al. [40] have assumed that the horizontal cross-section of a leg has a circular shape. However, this assumption does not hold in practice, for two reasons. Firstly, we have explained that the point cloud may be highly deformed, due to the sensors asynchronism, to the motion, and to noise. Secondly, in the swing phase, the sensors may see an horizontal cross-section of the foot instead of the lower part of the leg. Another kind of assumption often encountered in the literature is a maximal walking speed. Cui et al. have underlined the difficulties they encountered when the people are moving fast [11]. We have already observed walking speeds around $3.6 \mathrm{~m} / \mathrm{s}$ in medical applications where people are asked to walk in the "as fast as possible" paradigm [26]. Such a walking speed obviously violates the hypothesis encountered in most papers (eg $1.4 \mathrm{~m} / \mathrm{s}$ in [8] and $1.5 \mathrm{~m} / \mathrm{s}$ in [36]).

For all the reasons we have summarized here, we propose an alternative processing pipeline to the classical processing approach. The proposed pipeline is described in the next section.

\section{THE PROPOSED PROCESSING PIPELINE}

Figure 2 shows the proposed processing pipeline, which is organized in 7 steps. Some details are omitted for conciseness.

1. A background subtraction algorithm is applied on the distance profiles measured by each sensor, in order to filter out uninteresting static elements in the scene, as in $[9,13,16,34,37,41]$.

2. A polar to Cartesian coordinates transformation is applied on the distance profiles to convert the measures taken by each sensor into a point cloud.

3. Thanks to the calibration procedure [34], the individual point clouds are registered, and merged into a unique global point cloud.

4. A coarse tracking (eg using a KALMAN filter) is used to segment the person for which the feet trajectories need to be measured. The points corresponding to the other moving elements in the scene are filtered out. In some applications, a simple region of interest can be used in conjunction with (or in place of) this tracking. Or, one could also rely on human detection algorithms such as the one presented in [3].

5. Instead of clustering the remaining points into two blobs corresponding to the feet (which has been explained previously to be difficult in many cases), we localize the two feet directly from the point cloud of the person. To this end, we have designed an algorithm based on machine learning techniques. The model used for the prediction of the feet locations based on the point cloud is learned from synthetic samples randomly and automatically generated based on (1) the results of the calibration procedure [34], (2) a realistic walking person simulator, and (3) a software simulating RLSs. In this way, we avoid many sources of bias. For example, we do not make any assumption about the shape of the person's point cloud. The cloud deformation due to the sensors asynchronism and to the feet motion is properly handled, as well as the limited field of view of the sensors and the self-occlusions.

6. The labels "left" and "right" are given to the feet according to the result of an optimization taking multiple clues into account: the feet are never moving backwards significantly, only one foot is moving at a time, the left (right) foot turns around the right (left) one in the (counter-)clockwise direction during the swing phase, the feet trajectories are continuous, etc. Labeling errors are seldom, and only occur for very short periods of time.

7. We interpolate between the successive feet coordinates with the same labels. The resulting trajectories are filtered thanks to the knowledge of the noise affecting the output of the feet localizer (step 5, which has been previously evaluated).

Although this pipeline itself is innovative, new algorithms have been developed to implement the fifth and sixth steps. A detailed description of these algorithms is outside the scope of this paper. In addition, we plan to improve these two steps for next versions of GAIMS. Nevertheless, some experiments in the current configuration establish the relevance of our approach and the effectiveness of the new architecture (GAIMS provide very precise and accurate measures of the feet trajectories). This is highlighted by various achievements that have already been obtained with GAIMS in the medical field, as explained in the next section.

\section{RESULTS}

The feet trajectories measured and labeled by GAIMS can be used to derive many significant gait descriptors. At the time of writing, we have recorded, with GAIMS, the gait of 106 Healthy Persons (HP) and 71 Patients with Multiple Sclerosis (PMS) with the help of the university hospital of Liège (Belgium). Walking impairment is a good indicator of disease activity since it is frequent and appears early in the disease course of PMS. The volunteers were asked to walk in three modes (preferred pace, as fast as possible, and tandem gait) on a $25 \mathrm{ft}(7.62 \mathrm{~m})$ straight path, and on $\{1,5,25\}$ laps of a $20 \mathrm{~m} \infty$-shaped path. Many gait descriptors have been computed for each test and stored in a database (whose size is expected to grow significantly in the near future), which fed several studies. The main findings of those studies are summarized hereafter.

Several studies conducted by Phan-Ba et al. have demonstrated significant gait differences between HP and PMS [27, 29, 30], as well as between different categories of PMS defined either with respect to the Expanded Disability Status Scale (EDSS) [30] (this is 
S. Piérard, S. Azrour, and M. VAn Droogenbroeck. Design of reliable processing pipeline for the non-intrusive measurement of feet trajectories with lasers. In IEEE International Conference on Acoustics, Speech, and Signal Processing (ICASSP), Florence, Italy, pages 4432-4436, May 2014.

an composite score quantifying the disability of eight functional systems [20]) or with respect to the deceleration index [29] (this index is related to the motor fatigue [26]). More recently, also based on our database, Phan-Ba et al. have established that the diversity of gaits is mainly explained, for PMS, by two components: one is related to the walking speed and the other one to the ataxia and gait asymmetry [28].

One of the validation strategies we used for validating GAIMS showed that GAIMS is capable of detecting subtle within-subject gait modifications such as those induced by the intake of a low dose of alcohol $[32,33]$. These modifications are most probably related to ataxia. This opens new prospects concerning the follow up of PMS, since detecting gait modifications can be used for assessing the effectiveness of the medication and physical therapy.

Azrour [4] applied machine learning techniques on our database, and showed promising results concerning the diagnosis and follow up of PMS, based on the gait characteristics measured by GAIMS. He obtained an accuracy of $92 \%$ when distinguishing the HP from PMS. Moreover, he showed the possibility of predicting a score highly correlated with the EDSS based on the gait descriptors.

Giet [14] showed the existence of correlations between some gait characteristics measured with GAIMS and the quantity and quality of the physical therapy and physical activity followed by PMS. Note that only patients with an EDSS around 4 have been selected in his study; this corresponds to the ability to walk $500 \mathrm{~m}$ without aid. The significant gait characteristics include the speed, the double support time, the deviation from the followed path (during tandem walk), and the lateral distance between feet (an enlarged base of support improves the balance), as well as the speed decrease during a long walk (which is an estimation of the motor fatigue [26]). The positive correlation between the lateral inter-feet distance and the quantity of physical therapy and physical activity was unexpected and is still unexplained. Remarkably, correlations between some gait characteristics provided by GAIMS and the emotional state of the patients have also been observed by Giet [14]: the more the PMS feel coached by their physical therapist, the more the double support time is reduced when walking a small distance as fast as possible.

The findings of all those studies clearly show that our processing pipeline preserves the interesting (abnormal) components of the gait, which would be filtered out by the classical processing approach, as explained in Section 3. Moreover, the signal to noise ratio characterizing the gait descriptors is sufficiently high for allowing the detection of inter- and intra-subject gait modifications in medical applications. This validates the design and the processing pipeline currently used in GAIMS.

\section{CONCLUSION}

GAIMS is a powerful measuring system that provides reliable feet trajectories without requiring the observed person to wear any active or passive marker, sensor, etc. It is based on distance measures taken by range laser scanners, and it is therefore a non-intrusive system. This paper describes the challenges for obtaining reliable measures of the feet trajectories (precise, accurate, insensitive to the appearance of clothes and to the lighting conditions, etc), and proposes a new processing pipeline that is more effective than the traditional tracking paradigm. With our new processing algorithms, GAIMS has proven to be useful for medical applications. GAIMS could also be used for other applications requiring precise and accurate measures of the feet trajectories, such as the biometric identification.

\section{Acknowledgements}

We express our gratitude to all the volunteers who have accepted to be recorded with GAIMS. We thank the university hospital of Liège (www. chuliege.be), and the staff of neurology for both their help and availability. We are also grateful to the Walloon region of Belgium (www.wallonie.be) for funding this project and to BEA (www.bea.be) for the lasers and support. S. Azrour has a PhD grant of the "Fonds National de la Recherche Scientifique" (FNRS, Belgium, www. fnrs.be).

\section{REFERENCES}

[1] H. Acharya and R. Camicioli. The forgotten thesis of Gilles de la Tourette. University of Alberta Health Sciences Journal, 2(2):32-33, December 2005.

[2] K. Arras, S. Grzonka, M. Luber, and W. Burgard. Efficient people tracking in laser range data using a multi-hypothesis legtracker with adaptive occlusion probabilities. In ICRA, pages 1710-1715, Pasadena, May 2008.

[3] K. Arras, Ó. Mozos, and W. Burgard. Using boosted features for the detection of people in $2 \mathrm{D}$ range data. In ICRA, pages 3402-3407, Roma, Italy, April 2007.

[4] S. Azrour. Caractérisation des troubles de la marche par apprentissage automatique : détermination de scores adaptés à la sclérose en plaques à partir de données clinimétriques. Master's thesis, University of Liège, Belgium, 2013.

[5] S. Belachew, S. Piérard, R. Phan-Ba, and M. Van Droogenbroeck. Multimodal evaluation of gait and stride dynamics in relapsing and progressive forms of multiple sclerosis. Proceedings of the Belgian Royal Academies of Medecine, 1:66-69, 2012.

[6] N. Boulgouris, D. Hatzinakos, and K. Plataniotis. Gait recognition: a challenging signal processing technology for biometric identification. IEEE Signal Processing Magazine, 22(6):7890, November 2005.

[7] A. Carballo, A. Ohya, and S Yuta. Multiple people detection from a mobile robot using double layered laser range finders. In ICRA, Kobe, Japan, May 2009. Poster presented in the workshop "People Detection and Tracking".

[8] W. Chung, H. Kim, Y. Yoo, C.-B. Moon, and J. Park. The detection and following of human legs through inductive approaches for a mobile robot with a single laser range finder. IEEE transactions on industrial electronics, 59(8):3156-3166, August 2012.

[9] J. Cui, H. Zha, H. Zhao, and R. Shibasaki. Laser-based interacting people tracking using multi-level observations. In IROS, pages 1799-1804, Beijing, China, October 2006.

[10] J. Cui, H. Zha, H. Zhao, and R. Shibasaki. Robust tracking of multiple people in crowds using laser range scanners. In ICPR, volume 4, pages 857-860, Hong Kong, August 2006.

[11] J. Cui, H. Zha, H. Zhao, and R. Shibasaki. Laser-based detection and tracking of multiple people in crowds. CVIU, 106(23):300-312, May-June 2007.

[12] F. Faure, G. Debunne, M.-P. Cani-Gascuel, and F. Multon. Dynamic analysis of human walking. In 8th Eurographics Workshop on Computer Animation and Simulation, pages 53-65, Budapest, Hungary, September 1997. Springer. 
S. Piérard, S. AzRour, and M. VAN Droogenbroeck. Design of reliable processing pipeline for the non-intrusive measurement of feet trajectories with lasers. In IEEE International Conference on Acoustics, Speech, and Signal Processing (ICASSP), Florence, Italy, pages 4432-4436, May 2014.

[13] A. Fod, A. Howard, and M. Matarić. A laser-based people tracker. In ICRA, pages 3024-3029, Washington, USA, May 2002.

[14] A. Giet. Création et validation multimodale d'une échelle mesurant la qualité de la kinésithérapie et de l'activité physique chez les personnes souffrant de sclérose en plaques. Master's thesis, University of Liège, Belgium, 2013.

[15] D. Glas, T. Miyashita, H. Ishiguro, and N. Hagita. Automatic position calibration and sensor displacement detection for networks of laser range finders for human tracking. In IROS, pages 2938-2945, Taipei, Taiwan, October 2010.

[16] D. Hähnel, R. Triebel, W. Burgard, and S. Thrun. Map building with mobile robots in dynamic environments. In ICRA, volume 2, pages 1557-1563, Taipei, Taiwan, September 2003.

[17] M. Hashimoto, Y. Matsui, and K. Takahashi. People tracking with in-vehicle multi-laser range sensors. In Annual Conference of the Society of Instrument and Control Engineers (SICE), pages 1851-1855, Takamatsu (Kagawa University), Japan, September 2007.

[18] R. Kálmán. A new approach to linear filtering and prediction problems. Journal of Basic Engineering, 82(D):35-45, 1960.

[19] B. Kluge, C. Kohler, and E. Prassler. Fast and robust tracking of multiple moving objects with a laser range finder. In ICRA, volume 2, pages 1683-1688, Seoul, South Korea, May 2001.

[20] J. Kurtzke. Rating neurologic impairment in multiple sclerosis: an expanded disability status scale (EDSS). Neurology, 33(11):1444-1452, November 1983.

[21] J. Lee, T. Tsubouchi, K. Yamamoto, and S. Egawa. People tracking using a robot in motion with laser range finder. In IROS, pages 2936-2942, Beijing, China, October 2006.

[22] M. Lindner, I. Schiller, A. Kolb, and R. Koch. Time-ofFlight sensor calibration for accurate range sensing. CVIU, 114(12):1318-1328, 2010

[23] S. Mochon and T. McMahon. Ballistic walking. Journal of Biomechanics, 13(1):49-57, 1980.

[24] M. Mucientes and W. Burgard. Multiple hypothesis tracking of clusters of people. In IROS, pages 692-697, Beijing, China, October 2006.

[25] T. Pallejà, M. Teixidó, M. Tresanchez, and J. Palacín. Measuring gait using a ground laser range sensor. Sensors, 9:91339146, 2009.

[26] R. Phan-Ba, P. Calay, P. Grodent, G. Delrue, E. Lommers, V. Delvaux, G. Moonen, and S. Belachew. Motor fatigue measurement by distance-induced slow down of walking speed in multiple sclerosis. PLoS ONE, 7(4):8 pages, April 2012.

[27] R. Phan-Ba, G. Delrue, S. Piérard, E. Lommers, P. Calay, M. Van Droogenbroeck, and V. Delvaux. Influence of the mode of walk on walking speed in multiple sclerosis: are you walking comfortably? In Twenty-third meeting of the European Neurological Society, Barcelona, Spain, June 2013.

[28] R. Phan-Ba, S. Piérard, E. Lommers, G. Delrue, P. Calay, M. Van Droogenbroeck, V. Delvaux, and P. Maquet. Relative contribution of walking speed, ataxia and gait asymmetry to the composition of gait in multiple sclerosis. Multiple Sclerosis Journal, 19(S1):261, October 2013. Proceedings of ECTRIMS/RIMS 2013 (Copenhagen, Denmark), P608.
[29] R. Phan-Ba, S. Piérard, G. Moonen, M. Van Droogenbroeck, and S. Belachew. Deciphering distance-induced deceleration of gait and ataxia in people with multiple sclerosis. In Congress of the European Committee for Treatment and Research in Multiple Sclerosis (ECTRIMS), Lyon, France, October 2012. poster 755 .

[30] R. Phan-Ba, S. Piérard, G. Moonen, M. Van Droogenbroeck, and S. Belachew. Detection and quantification of efficiency and quality of gait impairment in multiple sclerosis through foot path analysis. In Congress of the European Committee for Treatment and Research in Multiple Sclerosis (ECTRIMS), Lyon, France, October 2012. poster 295.

[31] S. Piérard, S. Azrour, R. Phan-Ba, and M. Van Droogenbroeck. GAIMS: A reliable non-intrusive gait measuring system. ERCIM News, 95:26-27, October 2013.

[32] S. Piérard, R. Phan-Ba, V. Delvaux, P. Maquet, and M. Van Droogenbroeck. GAIMS: a powerful gait analysis system satisfying the constraints of clinical routine. Multiple Sclerosis Journal, 19(S1):359, October 2013. Proceedings of ECTRIMS/RIMS 2013 (Copenhagen, Denmark), P800.

[33] S. Piérard, R. Phan-Ba, and M. Van Droogenbroeck. Machine learning techniques to assess the performance of a gait analysis system. In European Symposium on Artificial Neural Networks, Computational Intelligence and Machine Learning (ESANN), Bruges, Belgium, April 2014.

[34] S. Piérard, M. Van Droogenbroeck, R. Phan-Ba, and S. Belachew. A new low-cost and non-intrusive feet tracker. In Workshop on Circuits, Systems and Signal Processing (ProR$I S C$ ), pages 382-387, Veldhoven, The Netherlands, November 2011.

[35] C. Premebida and U. Nunes. Segmentation and geometric primitives extraction from 2D laser range data for mobile robot applications. In 5th National Festival of Robotics (ROBOTICA), pages 17-25, Coimbra, Portugal, April-May 2005.

[36] D. Schulz, W. Burgard, D. Fox, and A. Cremers. Tracking multiple moving targets with a mobile robot using particle filters and statistical data association. In ICRA, volume 2, pages 1665-1670, Seoul, South Korea, May 2001.

[37] X. Shao, H. Zhao, K. Nakamura, R. Shibasaki, R. Zhang, and Z. Liu. Analyzing pedestrians' walking patterns using singlerow laser range scanners. In SMC, volume 2, pages 1202-1207, Taipei, Taiwan, October 2006.

[38] G. Taylor and L. Kleeman. A multiple hypothesis walking person tracker with switched dynamic model. In Australian Conference on Robotics and Automation, Canberra, Australia, December 2004.

[39] M. Teixidó, T. Pallejà, M. Tresanchez, M. Norgués, and J. Palacín. Measuring oscillating walking paths with a LIDAR. Sensors, 11:5071-5086, 2011.

[40] J. Xavier, M. Pacheco, D. Castro, A. Ruano, and U. Nunes. Fast line, arc/circle and leg detection from laser scan data in a player driver. In ICRA, pages 3930-3935, Barcelona, Spain, April 2005.

[41] H. Zhao and R. Shibasaki. A novel system for tracking pedestrians using multiple single-row laser-range scanners. IEEE Transactions on Systems, Man, and Cybernetics - Part A: Systems and Humans, 35(2):283-291, March 2005. 